\title{
Aluminum triggers broad changes in microRNA expression in rice roots
}

\author{
J.C. Lima ${ }^{1,2}$, R.A. Arenhart ${ }^{2}$, M. Margis-Pinheiro ${ }^{1,2}$ and R. Margis ${ }^{1,2,3}$ \\ ${ }^{1}$ Laboratório de Genomas e Populações de Plantas, Centro de Biotecnologia, \\ Universidade Federal do Rio Grande do Sul, Porto Alegre, RS, Brasil \\ ${ }^{2}$ Laboratório de Genética Molecular de Plantas, Departamento de Genética, \\ Universidade Federal do Rio Grande do Sul, Porto Alegre, RS, Brasil \\ ${ }^{3}$ Departamento de Biofísica, Universidade Federal do Rio Grande do Sul, \\ Porto Alegre, RS, Brasil \\ Corresponding author: R. Margis \\ E-mail: rogerio.margis@ufrgs.br
}

Genet. Mol. Res. 10 (4): 2817-2832 (2011)

Received January 12, 2011

Accepted March 9, 2011

Published November 10, 2011

DOI http://dx.doi.org/10.4238/2011.November.10.4

\begin{abstract}
MicroRNAs are small 21-nucleotide RNA molecules with regulatory roles in development and in response to stress. Expression of some plant miRNAs has been specifically associated with responses to abiotic stresses caused by cold, light, iron, and copper ions. In acid soils, aluminum solubility increases, thereby causing severe damage to plants. Although physiological aspects of aluminum toxicity in plants have been well characterized, the molecular mediators are not fully elucidated. There have been no reports about miRNA responses to aluminum stress. Modulation of miRNA expression may constitute a key element to explain the mechanisms implicated in aluminum toxicity and tolerance. We examined the expression of at least one miRNA member from each miRNA family in rice roots of Oryza sativa spp indica cv. Embrapa Taim and Oryza sativa spp japonica cv. Nipponbare under high concentrations of aluminum. Forty-six miRNA families were effectively detected by quantitative PCR. Among these, 13 were down-regulated and six were up-regulated in roots of the Nipponbare
\end{abstract}


cultivar after $8 \mathrm{~h}$ of aluminum treatment. In roots of the Embrapa Taim cultivar, five miRNAs were down-regulated and three were upregulated. Analyses of their putative targets suggest that these rice miRNAs are involved in the regulation of various metabolic pathways in response to high concentrations of aluminum.

Key words: Aluminum; MicroRNAs; Abiotic stress; Gene expression

\section{INTRODUCTION}

MiRNAs (microRNAs) are small 21-nucleotide-long RNA molecules with a central role in regulating gene expression (Xie et al., 2010). MicroRNAs act similarly to small interfering RNAs (siRNAs) by a mechanism of cleavage of their complementary mRNA targets or by repression of translation. The ancient origin of miRNAs, together with the potential link between miRNAs and development, implies that miRNAs might have participated in the origin and evolution of both plant and animal multicellular life (Reinhart et al., 2002). In plants, hundreds of miRNAs have been characterized by cloning and sequencing. Their putative targets, identified by in silico analysis, are mainly associated with developmental processes and stress responses (Chen et al., 2006). A large number of new miRNAs have been identified from in silico analyses of Arabidopsis thaliana, Populus trichocarpa, Brassica napus, and Oryza sativa (Lindow et al., 2007; Huang CF et al., 2010; Huang SQ et al., 2010) and by in-depth sequencing of small RNA libraries (Sunkar, 2010). In contrast to the discovery of many small RNAs, the biological role of the majority of miRNAs remains to be elucidated.

In plants, there are examples of miRNAs that regulate specific steps in cellular differentiation and organ development, while others have been associated with responses to biotic and abiotic stresses. The GAMYB-like gene, MYB33, which is important during anther development, is down-regulated by miRNA159-directed cleavage in Arabidopsis (Millar and Gubler, 2005). Post-transcriptional regulation of the flowering gene APETALA2, controlled by miRNA172 in Arabidopsis, incorporates the dual mechanism of mRNA cleavage and inhibition of translation (Zhu and Helliwell, 2011). Challenge of Arabidopsis with the flagellin protein contributes to Pseudomonas syringae resistance and has been associated with miRNA393a induction and repression of auxin signaling (Navarro et al., 2006). Also, in rice, miRNA393 and miRNA393b genes respond differently to salinity and alkaline stress. Arabidopsis cultivated under cold, high salt concentrations or with the plant hormone, abscisic acid, showed differential expression of several miRNAs (Jung and Kang, 2007). Increasing concentrations of sulfate repress the expression of miRNA395 and up-regulated the expression of its target ATP sulfurylase-1. Arabidopsis plants overexpressing miRNA395 result in strong down-regulation of ATP sulfurylases and sulfate transporters (Liang et al., 2010). MiRNA399 contributes to the maintenance of phosphate homeostasis by down-regulating the expression of ubiquitin conjugating enzyme (UBC) in plants cultivated under low inorganic phosphate conditions. Arabidopsis plants cultivated under high light intensity and high iron or copper concentrations down-regulate miRNA398 and up-regulate its target genes, superoxide dismutases AtSOD1 and AtSOD2, which play a role in the detoxification of reactive oxygen species (ROS). Moreover, new technologies for sequencing small RNA libraries have been showing a huge diversity of miRNAs regulating plant development and in response to biotic 
and abiotic stresses. These high-throughput methods for sequencing confirm the occurrence of conserved miRNAs and have accelerated the discovery of new small RNAs as major players in plant biology (Sunkar, 2010; Xie et al., 2010).

The previous examples make evident aspects about how miRNAs are involved in responses to biotic and abiotic stresses. While miRNAs are clearly involved in responses to metal stress caused by $\mathrm{Fe}$ and $\mathrm{Cu}$, there are presently no reports on miRNAs responding to aluminum (Al) treatment in plants. Al toxicity has been recognized as a major limiting factor in plant productivity on acidic soils. The primary Al toxicity symptoms occur in roots, causing the inhibition of the elongation process in the apical meristems (Kikui et al., 2005). An extensive review of the effects and mechanisms of $\mathrm{Al}$ tolerance point out some important features: i) low $\mathrm{pH}$ causes the solubilization of soil $\mathrm{Al}$; ii) impairment of root growth diminishes water and mineral uptake; iii) high $\mathrm{Al}$ reactivity could cause the interaction of this metal with many cellular sites; iv) Al can interact with some important $\mathrm{Ca}^{2+}$ signaling pathways, and v) cellular contact with $\mathrm{Al}$ can elicit ROS. A major gene conferring tolerance to Al, ALMT1, was cloned from wheat and also conferred Al tolerance in transgenic rice and tobacco cells. Differential display reverse transcription PCR has been used to identify genes that respond to $\mathrm{Al}$ in rice cultivars displaying different sensitivities to this metal. Among the genes identified, several were related to signal transduction, ion transport, cellular metabolism, stress, and cytoskeleton rearrangements (Yang et al., 2007). Two genes, OsSTAR1 encoding a nucleotide binding domain, and OsSTAR2, found to be a bacterial-type ABC gene, being expressed mainly in roots, conferred Al tolerance in rice. Also, in Arabidopsis, the ortholog of the OsSTAR2 gene is involved in $\mathrm{Al}$ detoxification, indicating a similar functional mechanism (Huang et al., 2010). Recently, a major protein, ART1, was proposed to be a transcriptional factor regulating several genes involved in $\mathrm{Al}$ response in rice, including OsSTAR1 and OsSTAR2 (Yamaji et al., 2009). The physiological aspects for $\mathrm{Al}$ resistance have been well studied, but the molecular regulatory mechanisms controlling the $\mathrm{Al}$ response are not completely known. In maize, a monocot plant species like rice, more genes were found in the Al-sensitive genotype than in the Al-tolerant genotype (Maron et al., 2008). However, a citrate-exclusion mechanism was suggested to release toxic Al in maize, which is known to be different for rice (Khan et al., 2009). Since miRNAs have only recently been shown to participate in the cellular regulatory machinery, and since the molecular aspects of plant-aluminum interactions are far from being elucidated, we examined the expression of mature miRNAs in roots of rice seedlings treated with $\mathrm{Al}$.

\section{MATERIAL AND METHODS}

\section{Plant material and growth conditions}

Rice plants cv. Nipponbare (O. sativa ssp japonica) and cv. Embrapa Taim (O. sativa ssp indica) were used in this study. Seeds were sown on Petri dishes and germinated for 4 days (48-h dark/48-h light cycle). Twelve-day-old plants were cultivated in a low-ionic-strength hydroponic medium (Baier et al., 1995), $\mathrm{pH}$ 4.5. The composition of the medium was $10 \mu \mathrm{M}$ $\left(\mathrm{NH}_{4}\right)_{2} \mathrm{SO}_{4} ; 400 \mu \mathrm{M} \mathrm{CaCl} 2.2 \mathrm{H}_{2} \mathrm{O} ; 250 \mu \mathrm{M} \mathrm{MgCl} 2.6 \mathrm{H}_{2} \mathrm{O} ; 40 \mu \mathrm{M} \mathrm{NH}_{4} \mathrm{NO}_{3} ; 650 \mu \mathrm{M} \mathrm{KNO}_{3}$. For the aluminum treatment, plants were cultivated with $450 \mu \mathrm{M} \mathrm{AlCl}_{3}$ for 4 and $8 \mathrm{~h}$. After treatment, the plants were harvested and the roots were separated from the aerial part and immediately frozen in liquid nitrogen for RNA extraction. 


\section{Quantitative real-time PCR (RT-qPCR)}

Total RNA from roots was extracted using the Trizol reagent, following manufacturer instructions (Invitrogen). A stem loop primer was used to synthesize the miRNA cDNAs (5'-GTCGTATCCAGTGCAGGGTCCGAGGTATTCGCACTGGATACGACNNNNNN$3^{\prime}$ ), where the letter $N$ represents the sequence of the six nucleotides that hybridizes to the $3^{\prime}$ end of the mature miRNA (Chen et al., 2005). Mature miRNAs cDNA was synthesized by adding 0.1 to $2 \mu \mathrm{g}$ total RNA; $100 \mathrm{U}$ MMLV reverse transcriptase (Promega); $0.05 \mu \mathrm{M}$ stem loop primer; $1 \mathrm{X}$ enzyme buffer; $0.33 \mathrm{mM}$ dNTP mix in a final volume of $30 \mu \mathrm{L}$. The cDNA synthesis conditions were $16^{\circ} \mathrm{C}$ for $30 \mathrm{~min}, 42^{\circ} \mathrm{C}$ for $30 \mathrm{~min}, 85^{\circ} \mathrm{C}$ for $5 \mathrm{~min}$. The RT-qPCR mix was $10 \mu \mathrm{L}$ of cDNA (1:2); $0.4 \mu \mathrm{L} 5 \mathrm{mM}$ dNTPs; $0.2 \mu \mathrm{L}$ of each forward (10 $\mu \mathrm{M})$ and universal $(10 \mu \mathrm{M})$ primers; $2 \mu \mathrm{L}$ 10X PCR buffer; $1.2 \mu \mathrm{L} \mathrm{MgCl}_{2} ; 2 \mu \mathrm{L} 1$ X SYBR Green (Molecular Probe); $0.1 \mu \mathrm{L}$ ROX (1:100 in water) (Invitrogen); $0.05 \mu \mathrm{L}$ Taq Platinum (Invitrogen) to a final volume of $20 \mu \mathrm{L}$. A forward primer (Table 1) and a universal reverse primer (5'-GTGCAGGGTCCGAGGT-3') were used in the quantitative PCR assays. The primers Os18SR (5'-ACACTTCACCGGACCATTCAA), Os 18SF (5'-CTACGTCCCTGCCC TTTGTACA); Os $S 27 \alpha \mathrm{R}$ (5'-ACGCCTAAGCCTGCTGGTT), Os $S 27 \alpha \mathrm{F}$ (5'-ACCACTTCGA CCGCCACTACT), OseF $\alpha 1 \mathrm{R}$ (5'-GACTTCCTTCACGATTTCATCGTAA), OseF $\alpha 1 \mathrm{~F}$ (5'-TT TCACTCTTGGTGTGAAGCAGAT); OsFDHR(5'-TTCCAATGCATTCAAAGCTG), OsFDHF (5'-CAAAATCAGCTGGTGCTTCTC); OssnU6R (5'-AGGGGCCATGCTAATCTTCT), and OssnU6F (5'-GGGGACATCCGATAAAATTG) were used as internal reference genes to normalize the expression of the miRNAs. The RT-qPCR conditions were $94^{\circ} \mathrm{C}$ for $5 \mathrm{~min}$ followed by 40 cycles at $95^{\circ} \mathrm{C}$ for $15 \mathrm{~s}, 60^{\circ} \mathrm{C}$ for $10 \mathrm{~s}, 72^{\circ} \mathrm{C}$ for $15 \mathrm{~s}$. Then, the samples were heated from $55^{\circ}$ to $99^{\circ} \mathrm{C}$ with an increase of $0.1^{\circ} \mathrm{C} / \mathrm{s}$ to acquire the denaturing curve of the amplified products. Relative quantifications of amplified products were made by the $2^{-\Delta \Delta C t}$ method (Livak and Schmittgen, 2001) using the Applied Biosystem 7500 Real-time PCR with the SDS software. SYBR-green (Molecular Probes) was used to detect amplification and estimate $\mathrm{C}_{\mathrm{T}}$ values and to determine specificity of amplicons by denaturing curves and melting temperatures $\left(\mathrm{T}_{\mathrm{m}}\right)$.

\section{In silico analysis of miRNA targets}

Putative miRNA targets were checked in silico using the miRU tool (Zhang, 2005). All miRNA sequences were retrieved from the MiRBase (http://microrna.sanger.ac.uk/). Based on the rules described to identify microRNA targets, we were able to find more than one putative target for some of the miRNAs. Although we could have missed real targets, we adopted the following stringent criteria to choose putative targets: a limit score of 3.0 for the kind of base pairs (Wooble and/or Watson Crick base pair) and a maximum of three mismatches between miRNAs and their putative targets (Brennecke et al., 2005).

\section{RESULTS}

In rice, several miRNA families have been described in silico. Among these, some families are represented by more than one gene member, and others are represented by only one gene member (Griffiths-Jones et al., 2006; Zhang et al., 2010). A PCR quantitative analysis was conducted to have a general view of miRNA expression in response to Al treatment. 
Table 1. List of forward primers used for the RT-qPCR analysis in rice roots.

\begin{tabular}{|c|c|}
\hline miRNA & Forward primer 5'-3' \\
\hline miRNA1561 & GCCCGCGACAGAAGAGAGTG \\
\hline miRNA159d & GGCCGGATTGGATTGAAGGGA \\
\hline miRNA160e & GGTGCCTGGCTCCCTGT \\
\hline miRNA162a & GGCCGGTCGATAAACCTCTGC \\
\hline miRNA164e & GGCTGGAGAAGCAGGGCA \\
\hline miRNA166k & GGCTCGGACCAGGCTTCA \\
\hline miRNA167a & GGCTGAAGCTGCCAGCAT \\
\hline miRNA168a & GCGTCGCTTGGTGCAGAT \\
\hline miRNA169g & GGCUAGCCAAGGAUGACUUGCCUA \\
\hline miRNA171a & GGTGATTGAGCCGCGCC \\
\hline miRNA172a & GGCCGGGAGAATCTTGATGATG \\
\hline miRNA390 & AAGCUCAGGAGGGAUAGCGCC \\
\hline miRNA393 & GGCGTCCAAAGGGATCGCA \\
\hline miRNA393b & GGCGTCCAAAGGGATCGCAT \\
\hline miRNA395a & GGU GAA GUG CUU GGG GGA ACU C \\
\hline miRNA396e & GGCCGTCCACAGGCTTTCTT \\
\hline miRNA397a & GGCCTCATTGAGTGCAGCG \\
\hline miRNA398a & GGCGGTGTGTTCTCAGGTCA \\
\hline miRNA398b & GGCGGTGTGTTCTCAGGTCG \\
\hline miRNA399d & UGCCAAAGGAGAGUUGCCCUG \\
\hline miRNA408 & CCGCUGCACUGCCUCUUC \\
\hline miRNA413 & GGCCCUAGUUUCACUUGUUCUGCAC \\
\hline miRNA414 & GGCCUCAUCCUCAUCAUCAUCGUCC \\
\hline miRNA415 & GGCCGCAACAGAACAGAAGCA \\
\hline miRNA416 & GGCCTGTTCGTCCGTACAC \\
\hline miRNA417 & GGGCCCGGAATGTAGTGAATTT \\
\hline miRNA418 & GGCCGGUAAUGUGAUGAUGAAAUGACG \\
\hline miRNA419 & GGCCGTGATGAATGCTGACG \\
\hline miRNA420 & GCGCGCTAAATTAATCACGGA \\
\hline miRNA426 & GCGCGGTTTTGGAAGTTTGTC \\
\hline miRNA435 & GGCCGUUAUCCGGUAUUGGAGUUGA \\
\hline miRNA437 & GGGCCGGAAAGTTAGAGAAGTT \\
\hline miRNA439 & GGTGTCGAACCGCGGTT \\
\hline miRNA440 & GGCAGTGTCTCCTGATGATCG \\
\hline miRNA441a & GGCCGGGTACCATCAATATAAATGT \\
\hline miRNA442 & GGCGUGACGUGUAAAUUGCGAG \\
\hline miRNA443 & GGCGGATCACAATACAATAAA \\
\hline miRNA444 & UUGCUGCCUCAAGCUUGCUGC \\
\hline miRNA445 & GGCCGGUAAAUUAGUGUAUAAACAUCCGAU \\
\hline miRNA446 & GGCCGCAUCAAUAUGAAUAUGGGAAAUGG \\
\hline miRNA528 & CCTGGAAGGGGCATGCA \\
\hline miRNA530 & GGCAGGTGCAGAGGCAGA \\
\hline miRNA531 & CTCGCCGGGGCTGCGTGC \\
\hline miRNA535 & GGCCCTGACAACGAGAGAGA \\
\hline miRNA806 & AUGUGCUAAAAAGUCAACGGUG \\
\hline miRNA807a & GGCCGTCATCTCACAGGT \\
\hline miRNA808 & GGCCGGATGAATGTGGGAAATG \\
\hline miRNA809a & GGCCGGTGAATGTGAGAAATGT \\
\hline miRNA811a & GGCCACCGTTAGATCGAGAAAT \\
\hline miRNA812 & GGCGACGGACGGTTAAACG \\
\hline miRNA813 & GGCGGGTTATGGAATGGGT \\
\hline miRNA814a & GGCCGGCACTTCATAGTACAAC \\
\hline miRNA815a & GGCCAAGGGGATTGAGGAG \\
\hline miRNA817 & GGCTCCAACTTGAGGCCC \\
\hline miRNA818a & GGCCGGCCAATCCCTTATATTATG \\
\hline miRNA819b & GGCCGUCAGGUUAUAAGACUUUCUAGC \\
\hline miRNA820a & GCGGCCTCGTGGATGG \\
\hline miRNA821a & GGCCGGGAAGTCATCAACAAAAAAG \\
\hline
\end{tabular}

MiRNAs recently identified in silico and by high-throughput methods were not included in this analysis (Zhang et al., 2010). The group composed of miRNA319, miRNA394, miRNA438, 
miRNA529, and miRNA816 was also excluded from analysis, since no putative mRNA target was found in the rice genome using the miRU tool.

An initial kinetics of $\mathrm{Al}$ effect on miRNA expression was provided by measuring mature miRNA393 levels in Nipponbare rice roots after 4 and $8 \mathrm{~h}$ of incubation with $450 \mu \mathrm{M} \mathrm{Al}$. miRNA393 was chosen because it has been identified in several plant systems and has been indubitably associated with plant responses to other abiotic stresses. The kinetics study was kept to a short 8-h time window to assure that direct effects of aluminum on miRNA expression would be evaluated, since long-term incubations could trigger secondary metabolic and structural effects that could also change miRNA levels. Analyses were focused on roots as they are the primary contact site with aluminum under natural field conditions. Also, roots are the organs where the toxic effects of $\mathrm{Al}$ are most obvious.

We found that miRNA393b expression was slightly down-regulated compared to control plants after $4 \mathrm{~h}$ of $\mathrm{Al}$ treatment. However, after $8 \mathrm{~h}$ a stronger down-regulation of miRNA393b was observed in response to Al in Nipponbare rice roots (Figure 1). Based on these results, the expression of all other miRNAs was evaluated after $8 \mathrm{~h}$ of Al treatment. We detected expression of 44 mature miRNAs. Among these, 13 were down-regulated and six were up-regulated in roots of rice seedlings treated with $450 \mu \mathrm{M} \mathrm{Al}$ for $8 \mathrm{~h}$ in a hydroponic culture under low $\mathrm{pH}$ (Figure 2). For the six up-regulated miRNAs, the level of expression varied from approximately 1.5 -fold to more than 2.0 -fold, that of miRNA528 being the highest (Figure 2). For the 13 down-regulated miRNAs, the level of expression was reduced approximately 50 to $95 \%$. Compared to control plants, miRNA415 expression was the most reduced in rice roots treated with $\mathrm{Al}$ for $8 \mathrm{~h}$ (Figure 2). As far as we know, this is the first time that expression of miRNA415 and miRNA426 has been detected. Moreover, they were differentially expressed in rice roots treated with $\mathrm{Al}$ for $8 \mathrm{~h}$ (Figure 2).

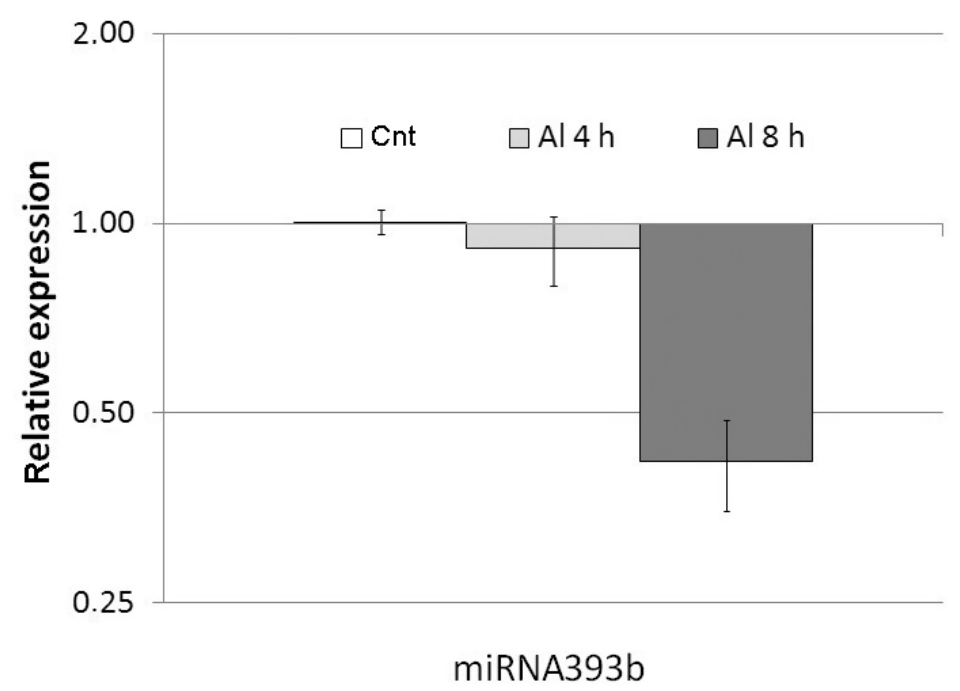

Figure 1. Quantification of mature miRNA393b in rice roots treated with aluminum (Al) for 4 and $8 \mathrm{~h}$ compared to control (Cnt). The histograms are means of three replicates. The values for the relative expression of miRNAs are expressed in logarithms of base 2. Expression of miRNA was normalized by comparison with expression of $O s 18 \mathrm{~S}$ and OssnU6 housekeeping RNAs. 


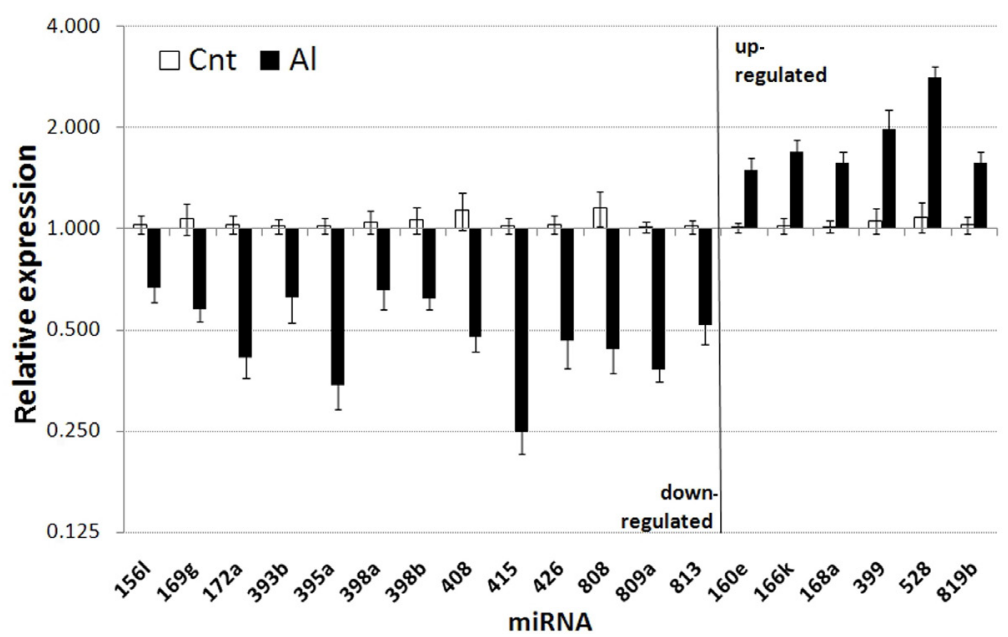

Figure 2. Comparative expression levels of miRNAs down- and up-regulated in Nipponbare rice roots after 8-h treatment with aluminum (Al) in respect to control (Cnt). The histograms are means of three replicates. The values for the relative expression of miRNAs are expressed in logarithms of base 2. Relative miRNA expression was normalized based on an average of five independent housekeeping genes and expressed on a logarithmic scale.

To compare microRNA expression pattern in rice roots of indica and japonica Nipponbare cultivars cultivated under high Al concentrations, we used Embrapa Taim, which is a Brazilian rice cultivar planted in waterlogged soils. For this analysis, we ran RT-qPCR only for the differentially expressed microRNAs found in the Nipponbare rice roots. We found that miRNA393b, miRNA395a, miRNA398a, miRNA398b, and miRNA408 were significantly down-regulated after $8 \mathrm{~h}$ of $\mathrm{Al}$ treatment (Figure 3). The expression of these miRNAs was

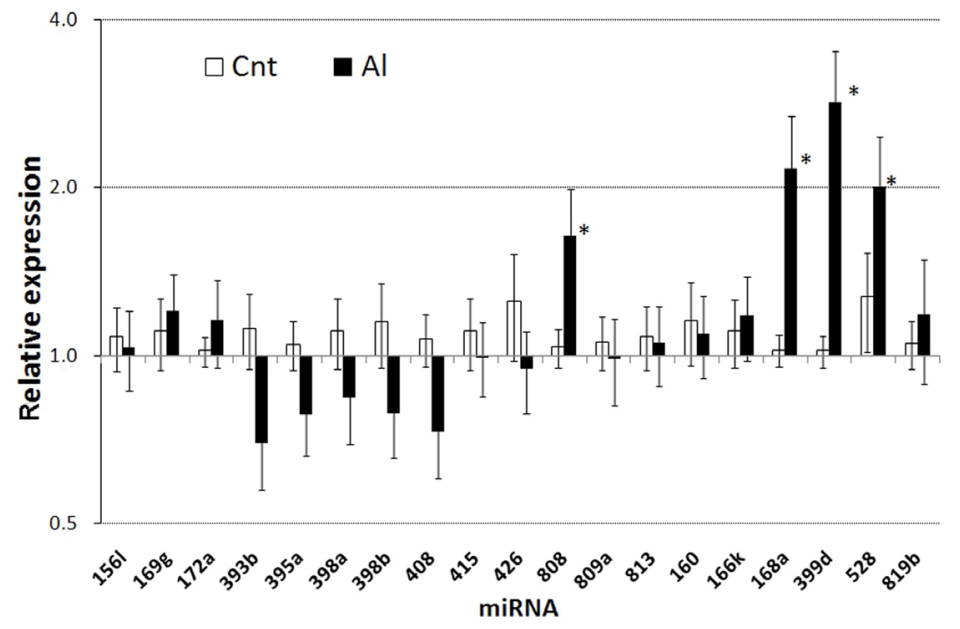

Figure 3. Comparative expression levels of miRNAs down- and up-regulated in Embrapa Taim rice roots after 8-h treatment with aluminum $(\mathrm{Al})$ in respect to control (Cnt). The histograms are means of four replicates. The values for the relative expression of miRNAs are expressed in logarithms of base 2. Relative miRNA expression was normalized based on an average of three independent housekeeping genes and expressed on a logarithmic scale. Asterisks correspond to miRNA with different expression levels between $\mathrm{Cnt}$ and $\mathrm{Al}(\mathrm{P}<0.05)$. 
reduced by $40 \%$. Only miRNA168a, miRNA399d, and miRNA528 were significantly upregulated, approximately 2.0 -fold after $8 \mathrm{~h}$ of Al treatment (Figure 3). To confirm the inverse correlation between the targets and the expression of the miRNAs, we ran an RT-qPCR for three miRNA528 targets; a slight down-regulation was observed for two of the targets (Figure 4 and Table 2).

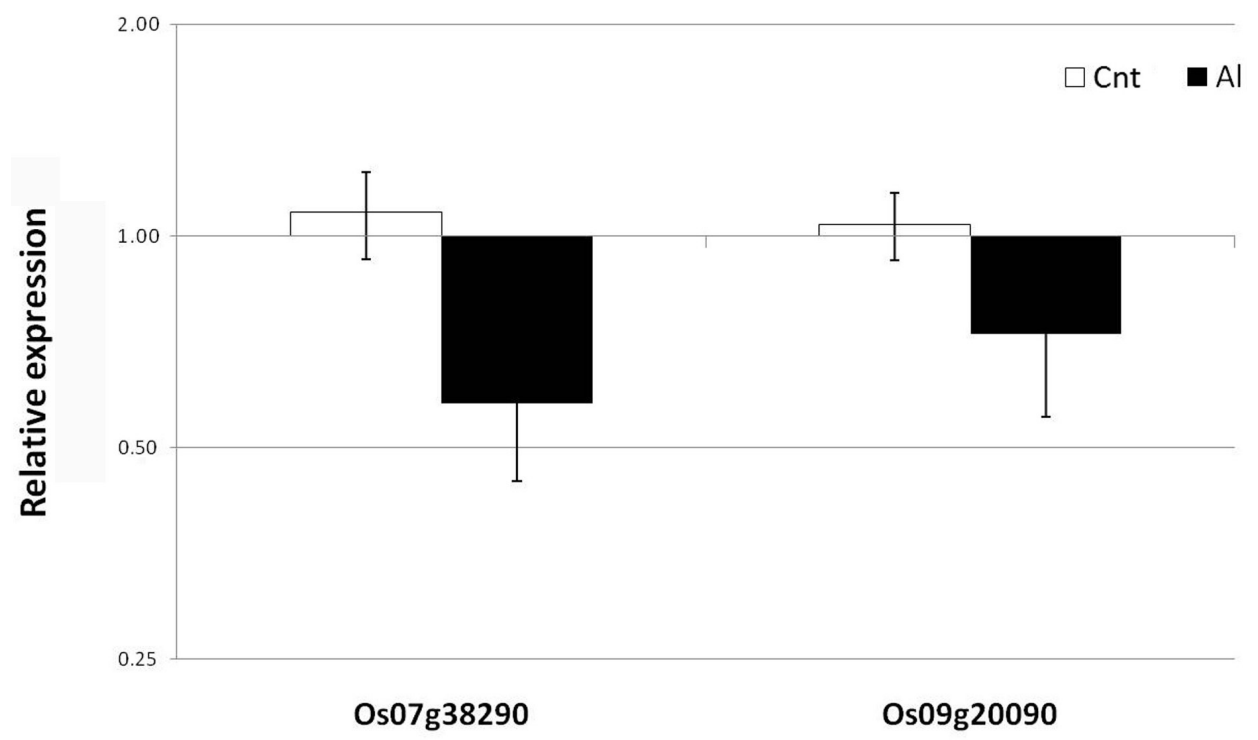

Figure 4. Comparative expression levels of miRNA528 targets in Embrapa Taim rice roots after 8-h treatment with aluminum $(\mathrm{Al})$ in respect to control $(\mathrm{Cnt})$. The histograms are means of four replicates. The values for the relative expression of miRNA targets are expressed in logarithms of base 2. Relative miRNA target expression was normalized based on an average of two independent housekeeping genes and expressed on a logarithmic scale.

Table 2. List of primers used for the target genes in rice roots.

\begin{tabular}{lll}
\hline Locus & Forward & Reverse \\
\hline Os07g38290 & GCCACCACTACTTCCTCTGC & GACGGAGAGCACGGACAC \\
Os09g20090 & TAACAAGCTCTGCAGCCTCA & CGACGTGAACTGGTAGCTGA \\
\hline
\end{tabular}

Identification of putative targets for rice miRNAs differentially expressed in response to $\mathrm{Al}$ treatment may contribute to our understanding of the involvement of post-transcriptional gene regulation in plant aluminum tolerance. In order to reduce the number of false-positive targets, we adopted a limit score of 3.0 for mismatches between the miRNA and its putative target and a limit score of 3.0 for the kind of base pair. Using the miRU tool to search for miRNA targets, we found at least one putative gene target for each miRNA (Tables 3 and 4). There are several genes involved in various cellular processes, like cell wall formation, stress responses, auxin signaling, and secondary metabolites. Recently, another study, confirmed by degradome sequencing, the cleavage of several rice miRNA targets, including some that we identified as putative ones in this report (Li et al., 2010). 


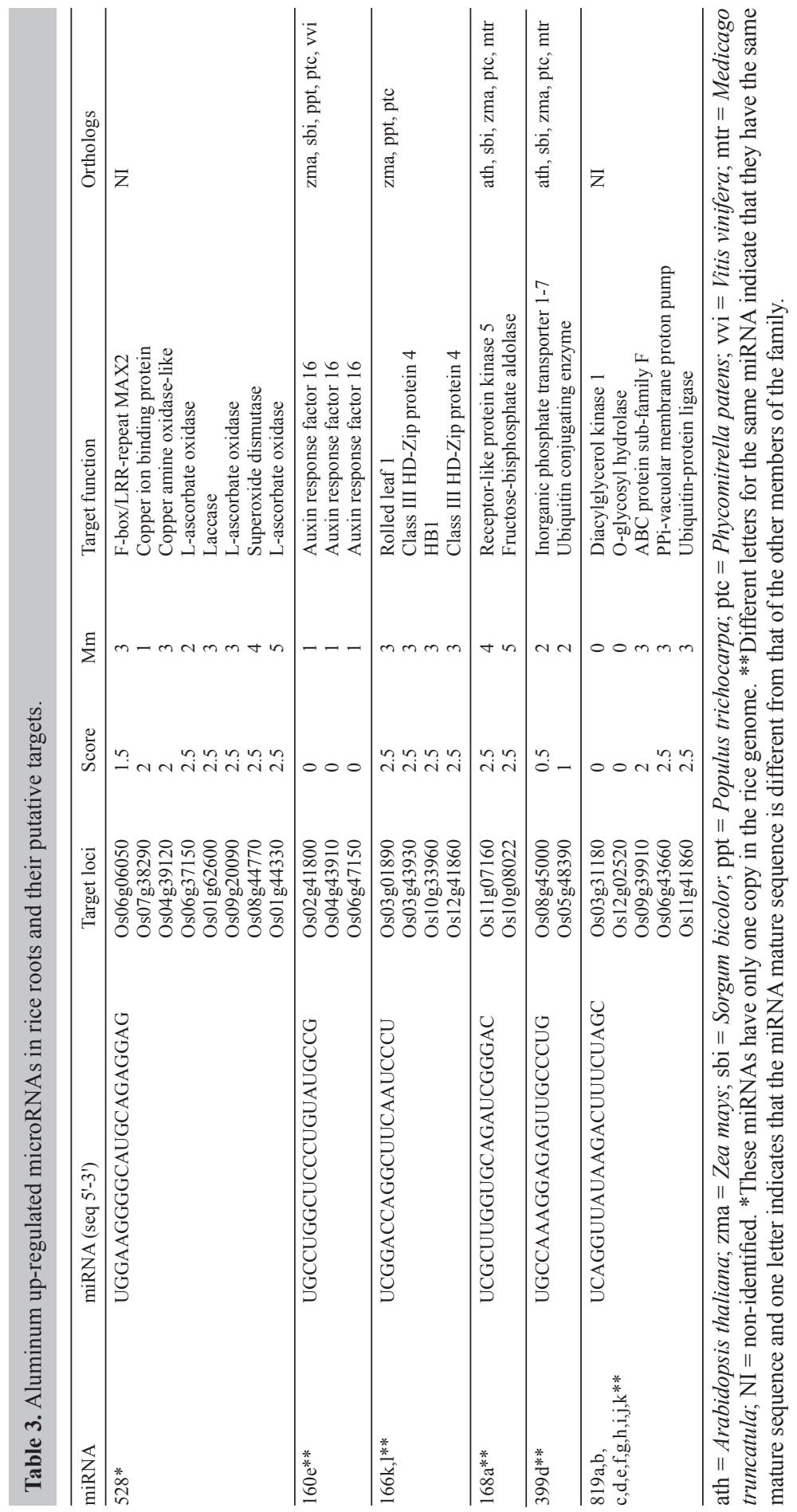


J.C. Lima et al.

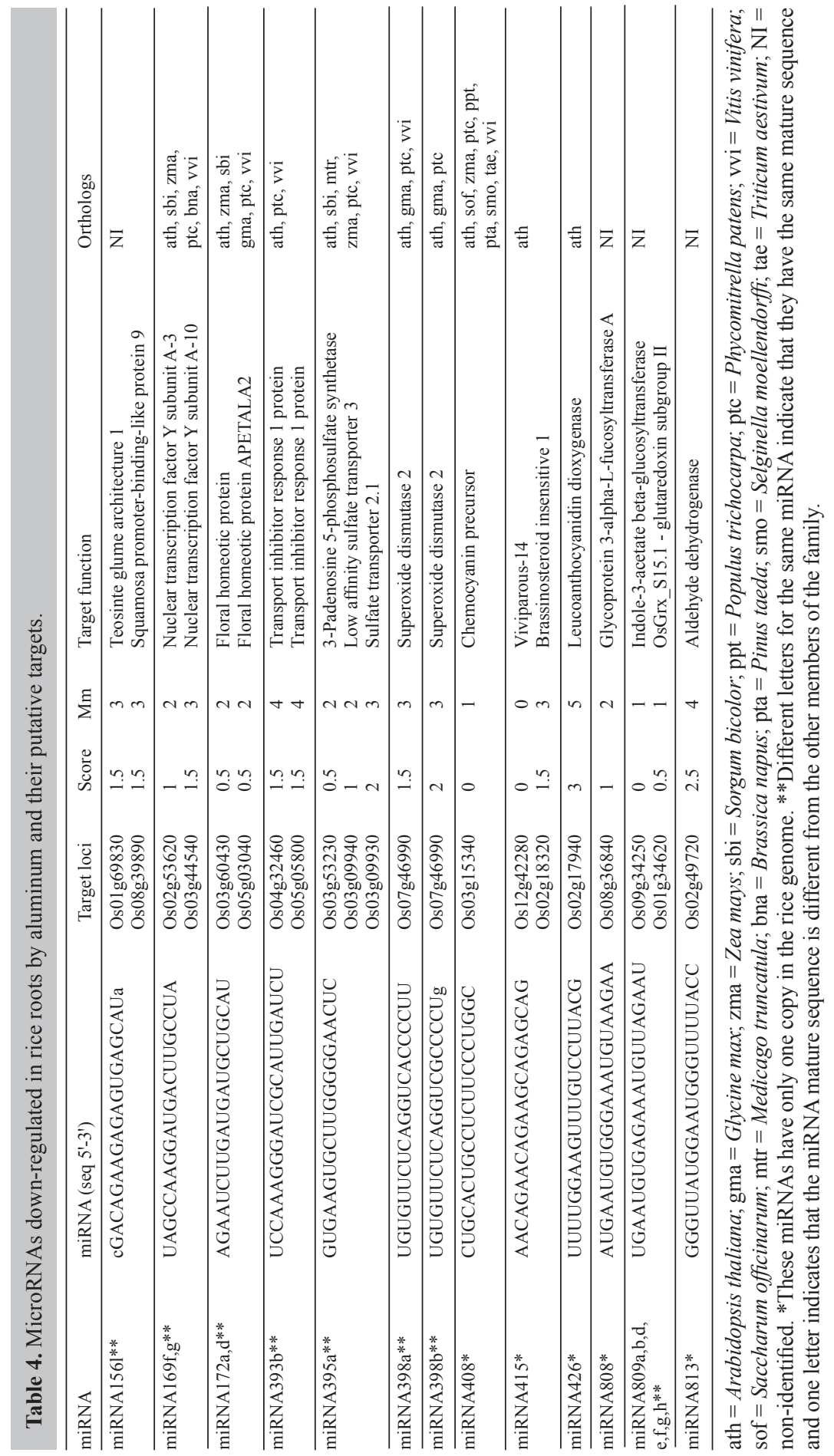




\section{DISCUSSION}

\section{Root growth responses}

Auxin is an important hormone involved in root development. The first symptom of $\mathrm{Al}$ stress in rice plants is root growth inhibition (Kikui et al., 2005). It has also been suggested that $\mathrm{Al}$ inhibits the basipetal transport of auxin in maize roots because exogenous applications of indole-3-acetic acid reverted this inhibition. In the japonica Nipponbare cultivar, miRNA393b down-regulated the TIR1 gene (Navarro et al., 2006). In Arabidopsis, TIR1 is an auxin-binding protein and mediates auxin signaling during lateral root formation by participating in protein degradation via SCF complexes (Xie et al., 2000). On the other hand, miRNA160e was up-regulated by Al treatment. Since miRNA160e regulates auxin response factors, it may be playing a counter-balancing role through the inverse regulation of miRNA393b in the auxin-signaling pathway in response to Al. miRNA528, which was found to be up-regulated in our experiment, has as a potential target an F-box/LRR repeat MAX2 gene. This gene encodes a protein that participates in an SCF complex that is involved in the regulation of shoot branching in Arabidopsis (Stirnberg et al., 2007). Also, it was suggested recently that another miRNA528 target, L-ascorbate oxidase, might have a role in regulating cell division during early developmental stages in rice seeds (Xue et al., 2009). MiRNA166k was also up-regulated. Our in silico analysis showed that this miRNA regulates genes from the III HD-ZIP family. In Arabidopsis, class III HD-ZIP genes are related to lateral root formation. It was also shown that miRNA166 is involved in the regulation of class III HD-ZIP genes in shoot apical meristem formation in Arabidopsis and in shoot meristem initiation in rice (Nagasaki et al., 2007). Hence, we suggest that up-regulation of miRNA528, miRNA160e, and miRNA166k, and down-regulation of miRNA393b could be contributing to fine control of root responses in rice plants in response to high concentrations of Al. Also, aluminum-induced stress can generate ROS, causing oxidized proteins to be degraded. Since miRNAs 528, 160e and 393b could be involved in the regulation of genes important in degradation pathways, these miRNAs may also play a role in protein quality control via the proteasome in response to $\mathrm{Al}$ stress.

The pectin methyl esters in cell wall polysaccharides have been found to be specifically involved in the exclusion of $\mathrm{Al}$ from the root apex. The fucosyl transferase gene is associated with the biosynthesis of cell wall sugars (Vanzin et al., 2002) and was identified as the primary target of miRNA808 (Table 4). The down-regulation of miRNA808 on Al-treated roots, associated with up-regulation of the fucosyl transferase gene, could cause structural modifications in the cells of rice plants (Figure 2).

\section{Metabolism of nutrients and carbon}

Mineral nutrients are essential for plant growth, and mineral deficiency can inhibit growth and damage the plants. Plants have evolved complex molecular mechanisms for cellular uptake, movement and metabolism of minerals (Grotz and Guerinot, 2002). In Arabidopsis, microRNAs such as miRNA399 and miRNA395 play a role in plant nutrient metabolism (Liang et al., 2010). MiRNA399d was up-regulated in our experiments. It regulates the PHO2 protein (UBC) that is involved in phosphate homeostasis in Arabidopsis. Northern blot and RT-qPCR analyses revealed elevated expression of members of the miRNA399 family, especially miRNA399d, in rice plants 
grown without inorganic phosphate (Bari et al., 2006). Because the primary symptom of Al stress is root growth impairment, causing inhibition of water and mineral uptake, accumulation of miRNA399d in rice roots treated with $\mathrm{Al}$ could be the reason for a lack of mineral uptake by the roots.

A proteomic approach in roots of rice seedlings grown under high $\mathrm{Al}$ and low phosphorus concentrations indicated alterations in carbon and nucleotide metabolism (Fukuda et al., 2007). These authors found that the gene encoding fructose bisphosphate aldolase, which is involved in carbon metabolism, was down-regulated $6 \mathrm{~h}$ after Al treatment. In our study, miRNA168a was up-regulated after $8 \mathrm{~h}$ of Al treatment. This suggests that miRNA168a could regulate the FBPA structural gene, its putative target.

MiRNA395 is reported to be a regulator of a low-affinity sulfate transporter and of ATP sulfurylases, key proteins in sulfur homeostasis (Liang et al., 2010). In roots of rice seedlings, expression of genes related to sulfur metabolism, including ATP sulfurylases, are up-regulated in response to Al (Yang et al., 2007). This response is possibly connected to down-regulation of miRNA395, since our analysis showed that miRNA395 expression was reduced after $8 \mathrm{~h}$ of $\mathrm{Al}$ treatment (Figures 2 and 3).

\section{Reactive oxygen species detoxification}

ROS are reactive radicals produced in aerobic cellular processes and can cause severe damage to cells. Superoxide dismutases (SOD) are important enzymes involved in detoxifying superoxide radicals $\left(\mathrm{O}_{2}^{-}\right)$, catalyzing their dismutation to hydrogen peroxide and $\mathrm{O}_{2}$. MiRNA398a and miRNA398b were down-regulated in response to Al treatment. It has already been shown that this family of microRNAs is involved in the regulation of SOD genes in Arabidopsis plants cultivated under high iron and copper concentrations. Under these stress conditions, increased expression of AtSOD1 and AtSOD2 genes is in contrast to the down-regulation of miRNA398. In rice, increased activity of SOD in seedlings treated with high Al concentrations was also found (Sharma and Dubey, 2007). Based on our results and those in the literature, miRNAs398a and b are involved in the regulation of SOD genes in plants under $\mathrm{Al}$ and other stress conditions.

\section{Secondary messengers and metabolites}

There is evidence that phosphatidic acid is an important lipid signaling molecule. In a suspension of cells of Coffea arabica, it was shown that Al blocks phosphatidic acid generation through the phospholipase C/diacylglycerol kinase route. We found up-regulation of miRNA819b, for which the diacylglycerol kinase gene is a putative target. We suggest that miRNA819b and Al have a synergistic effect in the phosphatidic acid pathway, or cellular responses positively regulate miRNA819b, which then down-regulates the diacylglycerol kinase gene.

It was found earlier that plantacyanins respond to abiotic stress in chickpea plants and subsequently that miRNA408 targets plantacyanins (Abdel-Ghany and Pilon, 2008). Based in our results, plus those in the literature, down-regulation of miRNA408 could also be playing a role in the response to $\mathrm{Al}$ stress in rice roots (Figures 2 and 3).

\section{Transcription factors}

It was recently reported that the plant nuclear factor $\mathrm{Y}$ has a crucial role in response to 
abiotic stress in maize, Arabidopsis, and rice (Zhao et al., 2007, 2009). Our analysis revealed that miRNA169g putatively targets genes from the nuclear factor Y family. Since miRNA169g is down-regulated in rice roots under Al treatment, this microRNA might have a role in increasing $\mathrm{Y}$ protein in rice roots under $\mathrm{Al}$ stress.

MiRNA1561 and miRNA172a are down-regulated in rice roots under Al treatment (Figures 2 and 5). In Arabidopsis, miRNA172 was characterized as an APETALA repressor gene in floral development. More detailed studies revealed that miRNA172 and miRNA156 play critical roles during plant development (Zhu and Helliwell, 2011).

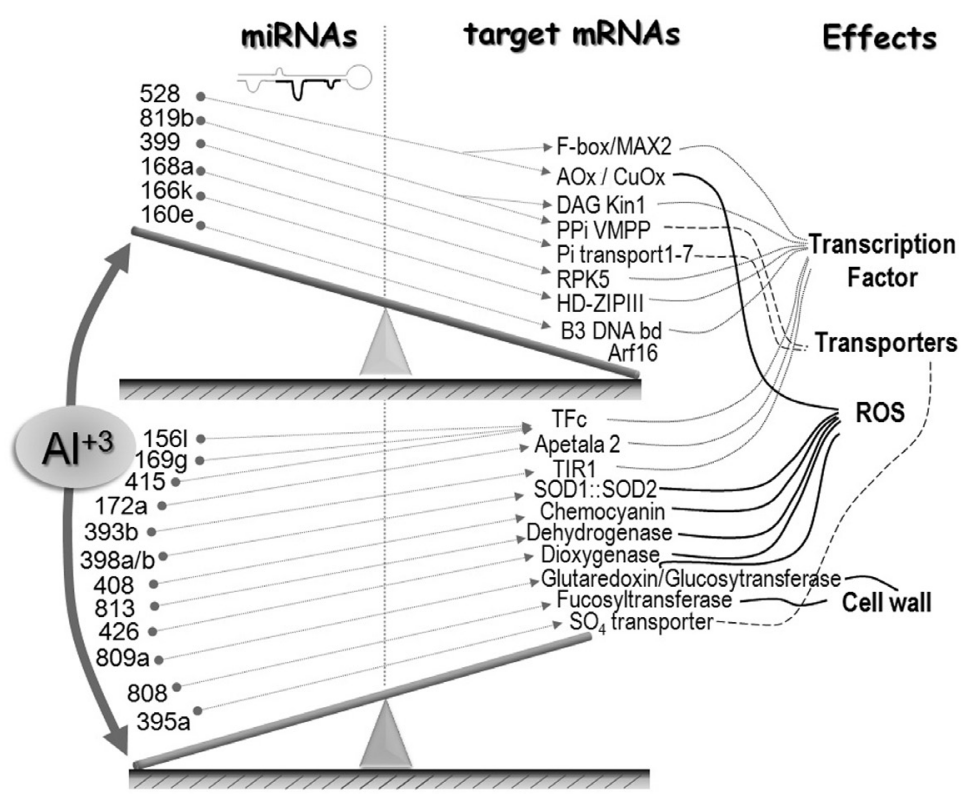

Figure 5. Diagram of the differentially expressed miRNAs in rice roots treated with aluminum (Al) and their putative targets. ROS $=$ reactive oxygen species.

\section{MiRNA responses to $\mathrm{Al}$ in Embrapa Taim}

Our results showed that for the indica cultivar, only 5 miRNAs were down-regulated compared to 13 found to be down-regulated in the japonica cultivar. Only three miRNAs were significantly up-regulated in the indica cultivar comparing to the six up-regulated in the japonica cultivar. Among the seven miRNAs differentially regulated in response to $\mathrm{Al}$ in the indica cultivar (Figure 3), six have been characterized as important regulators of stress responses (Lu and Huang, 2008). MiRNA395, a sulfur homeostasis regulator, is up-regulated in maize roots of both tolerant and sensitive lines under salt stress (Ding et al., 2009). Our analysis showed that miRNA395 is down-regulated in both indica and japonica varieties (Figures 2 and 3). Also, miRNA168, miRNA528 and miRNA399 were up-regulated in both rice varieties; it is known that these miRNAs respond to abiotic stresses (Xue et al., 2009). This pattern of expression could be explained by activation of similar pathways in response to Al stress in rice plants from different genetic backgrounds. It is suggested that the slight 
down-regulation of the miRNA528 target copper-ion gene, and especially of the L-ascorbate oxidase gene (Figure 4), results from the involvement of these proteins in the regulation of cell division (Xue et al., 2009). Since the primary symptom of sensitive plants treated with $\mathrm{Al}$ is root growth impairment, we suggest an initial arrest of cell divisions regulated by miRNA528 in rice roots. MiRNA408, which was also down-regulated in both rice varieties (Figure 2 and 3 ), is mediated by down-regulation of copper (Abdel-Ghany and Pilon, 2008). MiRNA393, which targets the gene that codes for the receptor of auxin TIR1, is undoubtedly important in the response to abiotic stresses in plants (Navarro et al., 2006). In the eudicot, Medicago truncatula, it was found that miRNA398 was down-regulated in response to Al, which is expected based on our results. MiRNA398 is known to be a regulator of genes that respond to abiotic stress in plants. A comparison between an Al-tolerant line and an Al-sensitive line in $M$. truncatula showed similar molecular responses related to genes involved in cell death, senescence, and cell wall degradation. Most Al-tolerant rice cultivars are derived from a japonica background, and Al-sensitive cultivars are derived from an indica background. Recently, it was found that several japonica cultivars with a different membrane lipid composition have a different tolerance response to toxic Al concentrations (Khan et al., 2009). This suggests that even in Al-tolerant rice cultivars, the responses are complex. Moreover, the different responses to $\mathrm{Al}$ among indica and japonica backgrounds are not yet well characterized.

Plant responses to abiotic stresses are quite complex and activate several mechanisms. $\mathrm{Al}$ is a special case in the study of plant-metal interaction, because Al toxicity in acidic soils worldwide can cause severe damage to important crops such as rice. On the other hand, the molecular mechanisms of plant-aluminum interaction are far from being fully elucidated. Our findings concerning miRNA differential expression in rice roots have revealed new components within the network of plant responses to abiotic stress caused by Al. In depth, sequencing of small RNA libraries from rice plants treated with $\mathrm{Al}$ will be useful to confirm and reveal new miRNAs and their targets in this complex plant-metal interaction.

\section{ACKNOWLEDGMENTS}

The authors thank Dr. J.C. Polacco for helpful discussion and critical reading of the manuscript. M. Margis-Pinheiro, and R. Margis were supported by grants from the Conselho Nacional de Desenvolvimento Científico e Tecnológico, CNPq, Brasil (\#308708/2006-7 and $\# 302684 / 2005-0)$. J.C. Lima is the recipient of a Ph.D. fellowship from CNPq. This study also received a specific Universal grant from CNPq (\#476005/2008-6).

\section{REFERENCES}

Abdel-Ghany SE and Pilon M (2008). MicroRNA-mediated systemic down-regulation of copper protein expression in response to low copper availability in Arabidopsis. J. Biol. Chem. 283: 15932-15945.

Baier AC, Somers DJ and Gustafson JP (1995). Aluminium tolerance in wheat: correlating hydroponic evaluations with field and soil performances. Plant Breed. 114: 291-296.

Bari R, Datt PB, Stitt M and Scheible WR (2006). PHO2, microRNA399, and PHR1 define a phosphate-signaling pathway in plants. Plant Physiol. 141: 988-999.

Brennecke J, Stark A, Russell RB and Cohen SM (2005). Principles of microRNA-target recognition. PLoS Biol. 3 : e85. Chen C, Ridzon DA, Broomer AJ, Zhou Z, et al. (2005). Real-time quantification of microRNAs by stem-loop RT-PCR. Nucleic Acids Res. 33: e179.

Chen Z, Zhang J, Kong J, Li S, et al. (2006). Diversity of endogenous small non-coding RNAs in Oryza sativa. Genetica 
128: 21-31.

Ding D, Zhang L, Wang H, Liu Z, et al. (2009). Differential expression of miRNAs in response to salt stress in maize roots. Ann. Bot. 103: 29-38.

Fukuda T, Saito A, Wasaki J, Shinano T, et al. (2007). Metabolic alterations proposed by proteome in rice roots grown under low $\mathrm{P}$ and high $\mathrm{Al}$ concentration under low pH. Plant Sci. 172: 1157-1165.

Griffiths-Jones S, Grocock RJ, van Dongen S, Bateman A, et al. (2006). miRBase: microRNA sequences, targets and gene nomenclature. Nucleic Acids Res. 34: D140-D144.

Grotz N and Guerinot ML (2002). Limiting nutrients: an old problem with new solutions? Curr. Opin. Plant Biol. 5: 158-163.

Huang CF, Yamaji N and Ma JF (2010). Knockout of a bacterial-type ATP-binding cassette transporter gene, AtSTAR1, results in increased aluminum sensitivity in Arabidopsis. Plant Physiol. 153: 1669-1677.

Huang SQ, Xiang AL, Che LL, Chen S, et al. (2010). A set of miRNAs from Brassica napus in response to sulphate deficiency and cadmium stress. Plant Biotechnol. J 8: 887-899.

Jung HJ and Kang H (2007). Expression and functional analyses of microRNA417 in Arabidopsis thaliana under stress conditions. Plant Physiol. Biochem. 45: 805-811.

Khan MS, Tawaraya K, Sekimoto H, Koyama H, et al. (2009). Relative abundance of Delta(5)-sterols in plasma membrane lipids of root-tip cells correlates with aluminum tolerance of rice. Physiol. Plant 135: 73-83.

Kikui S, Sasaki T, Maekawa M, Miyao A, et al. (2005). Physiological and genetic analyses of aluminium tolerance in rice, focusing on root growth during germination. J. Inorg. Biochem. 99: 1837-1844.

Li YF, Zheng Y, Addo-Quaye C, Zhang L, et al. (2010). Transcriptome-wide identification of microRNA targets in rice. Plant J. 62: 742-759.

Liang G, Yang F and Yu D (2010). MicroRNA395 mediates regulation of sulfate accumulation and allocation in Arabidopsis thaliana. Plant J. 62: 1046-1057.

Lindow M, Jacobsen A, Nygaard S, Mang Y, et al. (2007). Intragenomic matching reveals a huge potential for miRNAmediated regulation in plants. PLoS Comput. Biol. 3: e238.

Livak KJ and Schmittgen TD (2001). Analysis of relative gene expression data using real-time quantitative PCR and the 2(-Delta Delta C(T)) Method. Methods 25: 402-408.

Lu XY and Huang XL (2008). Plant miRNAs and abiotic stress responses. Biochem. Biophys. Res. Commun. 368: 458-462.

Maron LG, Kirst M, Mao C, Milner MJ, et al. (2008). Transcriptional profiling of aluminum toxicity and tolerance responses in maize roots. New Phytol. 179: 116-128.

Millar AA and Gubler F (2005). The Arabidopsis GAMYB-like genes, MYB33 and MYB65, are microRNA-regulated genes that redundantly facilitate anther development. Plant Cell 17: 705-721.

Nagasaki H, Itoh J, Hayashi K, Hibara K, et al. (2007). The small interfering RNA production pathway is required for shoot meristem initiation in rice. Proc. Natl. Acad. Sci. U. S. A. 104: 14867-14871.

Navarro L, Dunoyer P, Jay F, Arnold B, et al. (2006). A plant miRNA contributes to antibacterial resistance by repressing auxin signaling. Science 312: 436-439.

Reinhart BJ, Weinstein EG, Rhoades MW, Bartel B, et al. (2002). MicroRNAs in plants. Genes Dev. 16: 1616-1626.

Sharma P and Dubey RS (2007). Involvement of oxidative stress and role of antioxidative defense system in growing rice seedlings exposed to toxic concentrations of aluminum. Plant Cell Rep. 26: 2027-2038.

Stirnberg P, Furner IJ and Ottoline Leyser HM (2007). MAX2 participates in an SCF complex which acts locally at the node to suppress shoot branching. Plant J. 50: 80-94.

Sunkar R (2010). MicroRNAs with macro-effects on plant stress responses. Semin. Cell Dev. Biol. 21: 805-811.

Vanzin GF, Madson M, Carpita NC, Raikhel NV, et al. (2002). The mur2 mutant of Arabidopsis thaliana lacks fucosylated xyloglucan because of a lesion in fucosyltransferase AtFUT1. Proc. Natl. Acad. Sci. U. S. A. 99: 3340-3345.

Xie Q, Frugis G, Colgan D and Chua NH (2000). Arabidopsis NAC1 transduces auxin signal downstream of TIR1 to promote lateral root development. Genes Dev. 14: 3024-3036.

Xie Z, Khanna K and Ruan S (2010). Expression of microRNAs and its regulation in plants. Semin. Cell Dev. Biol. 21: 790-797.

Xue LJ, Zhang JJ and Xue HW (2009). Characterization and expression profiles of miRNAs in rice seeds. Nucleic Acids Res. 37: 916-930.

Yamaji N, Huang CF, Nagao S, Yano M, et al. (2009). A zinc finger transcription factor ART1 regulates multiple genes implicated in aluminum tolerance in rice. Plant Cell 21: 3339-3349.

Yang Q, Wang Y, Zhang J, Shi W, et al. (2007). Identification of aluminum-responsive proteins in rice roots by a proteomic approach: cysteine synthase as a key player in Al response. Proteomics. 7: 737-749.

Zhang Y (2005). miRU: an automated plant miRNA target prediction server. Nucleic Acids Res. 33: W701-W704.

Zhang Z, Yu J, Li D, Zhang Z, et al. (2010). PMRD: plant microRNA database. Nucleic Acids Res. 38: D806-D813.

Zhao B, Liang R, Ge L, Li W, et al. (2007). Identification of drought-induced microRNAs in rice. Biochem. Biophys. Res. 
Commun. 354: 585-590.

Zhao B, Ge L, Liang R, Li W, et al. (2009). Members of miR-169 family are induced by high salinity and transiently inhibit the NF-YA transcription factor. BMC Mol. Biol. 10: 29.

Zhu QH and Helliwell CA (2011). Regulation of flowering time and floral patterning by miR172. J. Exp. Bot. 62: 487-495. 